\title{
Copy number variation in Williams-Beuren syndrome: suitable diagnostic strategy for developing countries
}

\author{
Roberta L Dutra ${ }^{1,2,3^{*}}$, Rachel S Honjo ${ }^{1}$, Leslie D Kulikowski ${ }^{2}$, Fernanda M Fonseca ${ }^{3}$, Patrícia C Pieri ${ }^{3}$, \\ Fernanda S Jehee ${ }^{1}$, Debora R Bertola ${ }^{1}$ and Chong A Kim ${ }^{1}$
}

\begin{abstract}
Background: Williams-Beuren syndrome (WBS; OMIM 194050) is caused by a hemizygous contiguous gene microdeletion at 7q11.23. Supravalvular aortic stenosis (SVAS), mental retardation, and overfriendliness comprise typical symptoms of WBS. Although fluorescence in situ hybridization (FISH) is considered the gold standard technique, the microsatellite DNA markers and multiplex ligation-dependent probe amplification (MLPA) could be used for to confirm the diagnosis of WBS.

Results: We have evaluated a total cohort of 88 patients with a suspicion clinical diagnosis of WBS using a collection of five markers (D7S1870, D7S489, D75613, D7S2476, and D75489_A) and a commercial MLPA kit (P029). The microdeletion was present in 64 (72.7\%) patients and absent in 24 (27.3\%) patients. The parental origin of deletion was maternal in 36 of 64 patients (56.3\%) paternal in 28 of 64 patients (43.7\%). The deletion size was 1.55 $\mathrm{Mb}$ in 57 of 64 patients (89.1\%) and $1.84 \mathrm{Mb}$ in 7 of 64 patients (10.9\%). The results were concordant using both techniques, except for four patients whose microsatellite markers were uninformative. There were no clinical differences in relation to either the size or parental origin of the deletion.
\end{abstract}

Conclusion: MLPA was considered a faster and more economical method in a single assay, whereas the microsatellite markers could determine both the size and parental origin of the deletion in WBS. The microsatellite marker and MLPA techniques are effective in deletion detection in WBS, and both methods provide a useful diagnostic strategy mainly for developing countries.

\section{Background}

Williams-Beuren syndrome (WBS; OMIM 194050) is a neurodevelopmental disorder described independently $[1,2]$ as a syndrome involving facial appearance characteristics, supravalvular aortic stenosis (SVAS) and mental retardation. In fact, WBS presents a wide collection of symptoms affecting blood vessels, growth, intelligence, and behavior. Children with this condition have distinctive facial features, a hoarse voice associated with growth, mental retardation and an overfriendly personality; hyperacusis, infantile hypercalcemia, prematurely wrinkled skin are also common symptoms [3].

\footnotetext{
* Correspondence: roberta.dutra@icr.usp.br

'Department of Genetics, Instituto da Criança, Universidade de São Paulo, São Paulo, Brazil

Full list of author information is available at the end of the article
}

WBS is generally sporadic with frequency of approximately 1 in 7,500 live births with no ethnic or sex preference, although familial cases have been reported with apparent autosomal dominant inheritance $[4,5]$. Despite the consistency of the overall clinical features, the broad spectrum of anomalies and phenotypic variability frequently lead to a significant difference in the number of patients diagnosed [6].

WBS is caused by a hemizygous contiguous gene microdeletion of the WBS critical region on chromosome 7 at position $7 \mathrm{q} 11.23$. The most common deletion is found in $90 \%$ to $95 \%$ of WBS patients and spans a genomic region of approximately $1.55 \mathrm{Mb}$. It is the result of mispairing between the centromeric and medial LCR (Low copy repeats) blocks B (Bcen and Bmid) [7]. In $5 \%$ to $10 \%$ of cases, the breakpoints are within the centromeric and medial LCR blocks A (Acen and Amid)

\section{() Biomed Central}


and lead to an $\sim 1.84-\mathrm{Mb}$ deletion [8]. Atypical (approximately $0.2 \mathrm{Mb}$ to $\sim 2.5 \mathrm{Mb}$ ) deletions may be the leading cause of the substantial phenotypic variability among WBS patients [9].

Duplication of the WBS region occurs at half the frequency of deletions with less distinctive and somehow opposite clinical features, such as deficits of social interaction and an autistic-like phenotype [10,11].

Confirmation of clinical suspicion is essential for clinical monitoring of the patient and genetic counseling of the family. Although fluorescence in situ hybridization (FISH) is widely used and considered the gold standard for WBS molecular diagnosis, the use of microsatellite DNA markers has also been widely used and is considered highly informative and easily performed $[12,13]$.

Multiplex ligation-dependent probe amplification (MLPA) has been introduced into DNA diagnostic laboratories for the detection of deletions and/or duplications in several disease genes [14]. MLPA kit for WBS, makes possible a more precise mapping of the deletion in the critical region, compared with the FISH [15]. In this study, the results obtained with microsatellite markers were compared with those obtained with MLPA.

It can be argued that both techniques, together, are extremely valuable tools for the diagnosis of the WBS patients and that the implementation of both methods should be considered.

\section{Results}

A total of 88 patients with the suspicion of a clinical diagnosis of WBS were tested. The five markers (D7S1870, D7S489, D7S613, D7S2476 and D7S489A) were informative in 84 patients and not informative in 4 patients. The most informative marker was D7S1870 (78.4\% of patients), followed by D7S613 (68.2\% of patients), D7S489 (65.9\% of patients) and D7S2476 (57.9\% of patients). The microdeletion was present in 64 (72.7\%) patients and absent in $24(27.3 \%)$ patients.

The observed deletion size was $1.55 \mathrm{Mb}$ in 57 of 64 patients $(89.1 \%$ ) and $1.84 \mathrm{Mb}$ in 7 of 64 patients $(10.9 \%)$. For the parental origin, the deletion was maternal in 36 of 64 patients (56.3\%) and paternal in 28 of 64 patients (43.7\%).

Using the MLPA kit (P029), the results were concordant with the microsatellite marker analysis in 84 patients and on 4 cases the deletion was only detected by MLPA (Figure 1). FISH was performed in all patients and the results were concordant with those found by microsatellites and MLPA.

The microsatellite markers used in the present study, are located in different regions in comparison with the probes in the P029 kit for WBS (Figure 2). Except the D7S489 marker and the FZD9 probe from MLPA P029

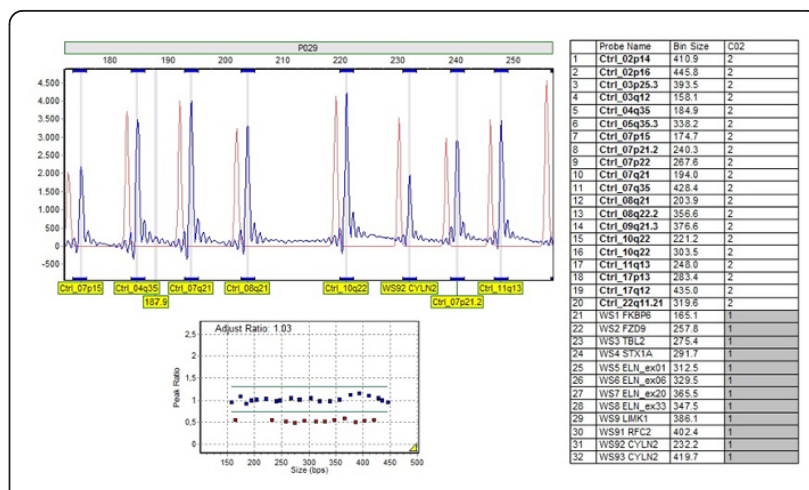

Figure 1 Genotyping by MLPA technique (SALSA kit P029) using the software GeneMarker ${ }^{\circledR}$ for analysis. Hemizygous contiguous gene microdeletion, can be visualized by probes 21 to 32. With the presence one copy these genes in the WBS critical region, $7 q 11.23$.

kit that are in the same position (Figure 3). Considering both techniques, there was no clinical difference in relation to either the size of deletion or the parental origin of deletion.

\section{Discussion}

Microsatellite DNA markers and MLPA have been considered highly informative and easily manageable for diagnostic confirmation of WBS.

In our study, five microsatellite markers (D7S1870, D7S489, D7S613, D7S2476, and D7S489A) were informative, except in four cases.

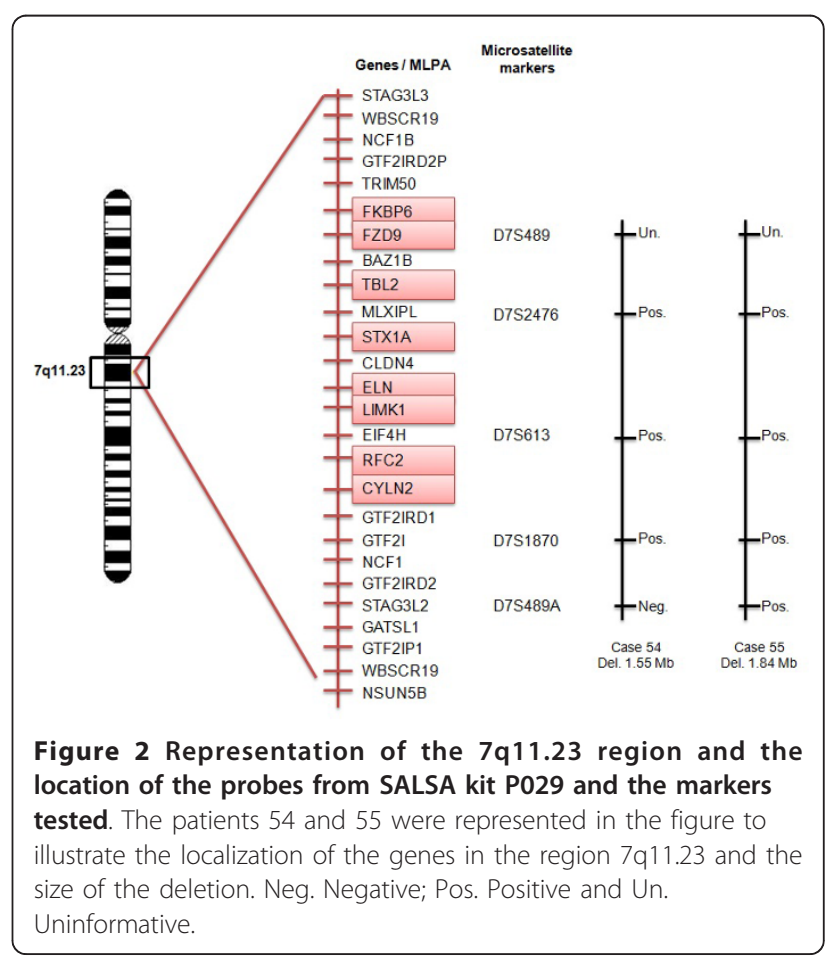



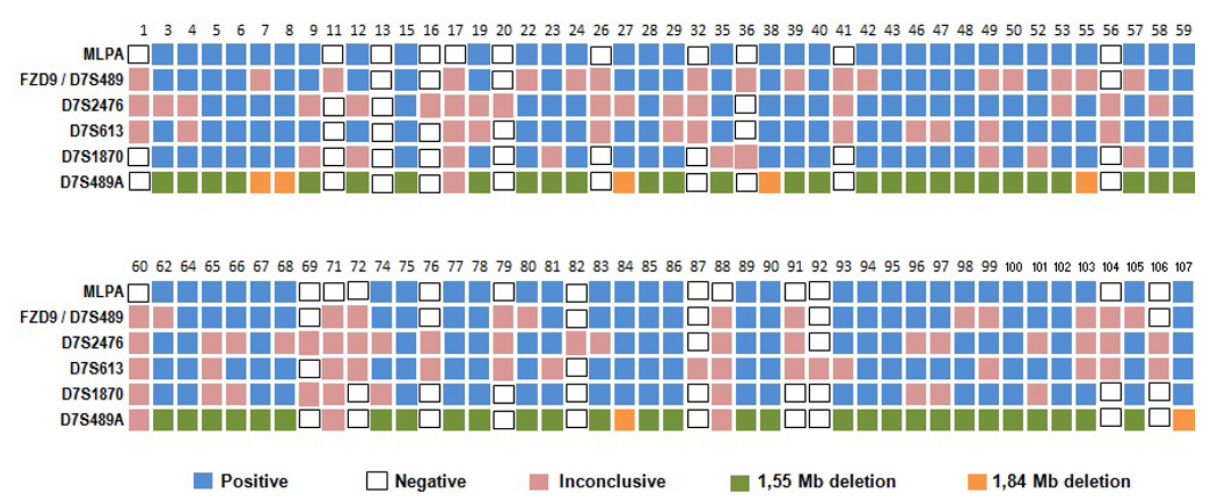

Figure 3 Comparison between the results obtained by microsatellite markers and MLPA. A total of 88 patients participated of the study and numbered 1 to 107. The correspondent probe to gene FZD9 is localized in the same region that D7S489 microsatellite marker.

However, in these particular cases, short stature, microcephaly, and cardiovascular anomalies were absent, but not in one patient that presented mitral and tricuspid regurgitation and hiperacusis.

The D7S1870 microsatellite marker showed the highest power of detection, able to identify $78.4 \%$ of the cases by itself, which confirmed the results from previous studies [8,16-19].

Two best markers (D7S1870 and D7S613) in our study were able to detect the deletion in $93.2 \%$ of cases when used together. When the D7S613 and D7S489 markers were included, informative detection increased to almost $95 \%$.

The microsatellite marker D7S489A was effective in the analysis of deletion size. The $1.55-\mathrm{Mb}$ deletion was found in 57 of 64 (89.1\%) patients, and the 1.84-Mb deletion was found in 7 of 64 patients (10.9\%); these observed percentages are similar to those found in other studies in the literature [8].

Using markers to identify the parental origin, we found no significant difference between the frequencies of maternal and paternal deletions $(56.3 \%$ and $43.7 \%$, respectively), and the literature is concordant with our findings $[12,13,17,19]$.

There was also no relationship between clinical features with the size of the deletion and with the parental origin.

Since the MLPA technique was developed [14], it has been tested as a diagnostic method in several diseases involving chromosomal disorders. In this study, we used the MLPA kit (P029) to observe the microdeletion in 64 $(72.7 \%)$ patients and find it was absent in $24(27.3 \%)$ patients.

We find four discrepant results comparing the microsatellite markers and the MLPA method in the detection of deletions in the WBS critical region. In these patients where the microsatellite markers are uninformative, detection of the deletion can be confirmed using the
MLPA technique. These patients present a phenotypic variability that often leads to diagnostic difficulties and the confirmation of results only was possible using MLPA technique.

The microsatellite markers were efficient in deletion detection for WBS when compared to the MLPA. They allowed for the detection of deletions larger than 1.55 $\mathrm{MB}$ and for detection of the parental origin of the deletion.

FISH is widely used and considered the gold standard for WBS molecular diagnosis, however, FISH is laborintensive, time-consuming, and it does not allow the detection of the exact size of the deletion [20].

The cost of the microsatellite marker technique has greatly decreased, and it can be deployed in molecular biology laboratories that have basic equipment for conventional PCR reactions and a vertical electrophoresis system.

The most important advantages of the MLPA are its relative simplicity, low cost, rapid turnaround ( 2 days), ease of multiplexing to permit high confidence in the results, high accuracy of copy number estimation, and the potential for combination of copy number analysis with other applications, such as methylation detection or SNP genotyping [21].

The accuracy of both diagnostic tests is well recognized to be susceptible to technical problems and clinical heterogeneity. In our study, FISH, markers and MLPA presented higher sensitivity (99.8\%), similar to others studies [22] and microsatellites markers presents lower specificity compared to FISH and MLPA (93\%).

Real-time quantitative polymerase chain reaction technique (QPCR) and array-based comparative genomic hybridisation (array-CGH) are also being used for the molecular diagnosis of WBS.

QPCR is considered a robust methodology, with easy interpretation, and simple to set up $[23,24]$. Conversely, to perform this technique we need sophisticated 
equipments and specific primers for each target region, differently from MLPA, where the simultaneous hybridization of more than 40 different probes can be used in one single reaction.

Recently, array-CGH has also been proved also to be a powerful and promising method to detect microdeletions and to identify novel cytogenetic abnormalities [25]. However, the resolution of array-CGH can vary depending on the format and design of the array [26]. Additionally, this method is relatively difficult and costly, and it requires a different setup as far as instrumentation is concerned [25].

Economic models are important to help health professionals to take decisions based on available strategies. The molecular tests available together with socio economic characteristics of the country is fundamental when a new strategy is considered to be taken, especially in developing countries where resources are limited [27].

\section{Conclusions}

The diagnosis of WBS based on clinical assessments may be difficult because of the great variability of its manifestations. Laboratory tests to detect the microdeletions in $7 q 11.23$ are essential to confirm the clinical diagnosis of WBS.

In summary, the microsatellite marker and MLPA techniques are effective in deletion detection in WBS, and both methods improve complete molecular coverage in screening of the critical region mainly for developing countries.

\section{Methods}

\section{Subjects}

A total cohort of 88 patients with a clinical diagnosis of WBS (56 boys and 32 girls) were followed through clinical evaluation by geneticists of the Unit of Clinical Genetics - Instituto da Criança, Hospital das Clínicas Universidade de São Paulo (ICr-HCFMUSP), Brazil. The inclusion criteria were dysmorphic facial features suggestive of WBS and the presence of cardiovascular disorders, mainly SVAS.

The study was approved by the Institutional Review Board - Ethics Committee for Analysis of Research Projects HCFMUSP/Cappesq - and written consent was obtained from all participants.

Among the 88 patients, DNA from both parents was obtained in 80 cases; in 8 cases, the molecular analysis was performed only with maternal DNA. Most of patients had normal GTG band karyotype and FISH was previously had been done in 24 patients.

The molecular study was performed in Laboratory of Genomic Pediatrics - LIM 36 - (Icr -HCFMUSP). DNA was isolated from peripheral blood lymphocytes using a salt precipitation technique [28].

\section{Microsatellite markers}

The five microsatellites markers used included D7S1870, D7S489, D7S613 and D7S2476 inside the common 1.55$\mathrm{Mb}$ deletion and D7S489A to distinguish deletions of 1.84 $\mathrm{Mb}$. PCR reactions were carried out according to Dutra et al. (2011) [13].

Patient genotypes were compared with those of their parents. Deletions were diagnosed as maternal when the proband presented with gel bands representing the allelic marker inherited only from the father. When by chance both parents have the same alleles, the monoallelic inheritance of the corresponding microsatellite marker by the proband indicated an uninformative result.

We first used a two-step algorithm to identify the most common 1.55-Mb deletion. We then tested the D7S489A marker either to identify the larger $1.84-\mathrm{Mb}$ deletion (in those patients in which a deletion of at least one marker was detected in the first step) or to confirm the lack of a deletion.

\section{MLPA}

The MLPA (SALSA kit P029 - MRC-Holland, Amsterdam, The Netherlands) containing probes for eight genes from the WBS critical region (FKBP6, FZD9, TBL2, STX1A, ELN, LIMK1, RFC2 and CYLN2) were used. The ELN and CYLN2 probes for various exons are present in the kit. Denaturation, overnight hybridisation, ligation and PCR were performed according to the manufacturer's instructions.

MLPA products were separated on a MegaBACE ${ }^{\mathrm{TM}}$ 1000 (GE Life Sciences, Waltham, USA) using MegaBACE ET SIZE Standards ET550-R (GE Life Sciences, Waltham, USA). The analysis was performed using the GeneMarker, version 1.6, software (Softgenetics, State College, PA, USA). The ratio of the probes' peak heights was determined by comparing the probes' peak heights obtained from patient samples to those obtained from three normal control samples.

\section{Statistical analysis}

Pairwise comparisons between clinical features of WBS and the presence of deletion, clinical features and deletion size and clinical features and parental origin of deletion were tested for significance using two-tailed Fisher's exact test. A $2 \times 2$ contingency table was used to compare clinical features. $\mathrm{P}$ analysis was performed in SPSS 13.0 software and considered statistically significant when $\mathrm{p} \leq 0.05$.

\section{Acknowledgements}

The authors are grateful to Dr. Ulysses Dória-Filho (Nucleo de Apoio Metodológico do Instituto da Criança - FMUSP) for his help with the statistical analysis and to Dra. Juliana Forte Mazzeu de Araújo with technical 
assistance in the MLPA technique. This work was supported by FAPESP grants $n^{\circ}$ 2008/55391-6, grants $n^{\circ}$ 2009/53105 and CNPq grants $n^{\circ} 401910$ / $2010-5$

\section{Author details}

${ }^{1}$ Department of Genetics, Instituto da Criança, Universidade de São Paulo, São Paulo, Brazil. Department of Pathology, LIM 03, Universidade de São Paulo, São Paulo, Brazil. ${ }^{3}$ Laboratory of Genomic Pediatrics - LIM 36, Instituto da Criança, Universidade de São Paulo, São Paulo, Brazil.

\section{Authors' contributions}

RLD graduate student (PhD), involved in drafting the manuscript, participated in the design of the study and collaborated with analysis and interpretation of data. RSH Medical and graduate student (PhD) participated in the design of the study and collaborated with updating medical records and ambulatory care of Williams syndrome patients. LDK Biologist PhD, sponsor of Cytogenomics group, participated in the design of the study and collaborated with final review of the manuscript. FMF Technical laboratory LIM 36, collaborated with the microsatellites markers experiments. PCP Biologist in LIM 36, collaborated with the standardization of methods (microsatellite markers) and with interpretation of data. FSJ Biologist PhD, collaborated with the standardization of methods (MLPA) and with analysis and interpretation of data. DRB Assistant Doctor in Unit of Genetics, Instituto da Criança, FMUSP, collaborated with the ambulatory care of Williams syndrome patients. CAK Sponsor of the research of this work and responsible for Unit of Genetics, Instituto da Criança, FMUSP and participated with revising it critically for important intellectual content. All authors read and approved the final manuscript.

\section{Competing interests}

Non-financial competing interests.

Received: 12 October 2011 Accepted: 9 January 2012

Published: 9 January 2012

\section{References}

1. Williams JC, Barratt-Boyes BG, Lowe JB: Supravalvular aortic stenosis. Circulation 1961, 24:1311-1318.

2. Beuren AJ, Apitz J, Harmjanz D: Supravalvular aortic stenosis in association with mental retardation and a certain facial appearance. Circulation 1962, 26:1235-1240.

3. Morris CA, Demsey SA, Leonard CO, Dilts C, Blackburn BL: Natural history of Williams syndrome: physical characteristics. J Pediatr 1988, 113:318-326.

4. Strømme P, Bjørnstad PG, Ramstad K: Prevalence estimation of Williams syndrome. J Child Neurol 2002, 17:269-271.

5. Morris CA, Thomas IT, Greenberg F: Williams syndrome: autosomal dominant inheritance. Am J Med Genet 1993, 47:478-481.

6. Ashkenas J: Williams syndrome starts making sense. Am J Hum Genet 1996, 59:756-761.

7. Peoples R, Franke Y, Wang YK, Pérez-Jurado L, Paperna T, Cisco M, Francke U: A physical map, including a BAC/PAC clone contig, of the Williams-Beuren syndrome-deletion region at 7q11.23. Am J Hum Genet 2000, 66:47-68

8. Bayés M, Magano LF, Rivera N, Flores R, Pérez Jurado LA: Mutational mechanisms of Williams-Beuren syndrome deletions. Am J Hum Genet 2003, 73:131-151.

9. Gagliardi C, Bonaglia MC, Selicorni A, Borgatti R, Giorda R: Unusual cognitive and behavioural profile in a Williams syndrome patient with atypical 7q11.23 deletion. J Med Genet 2003, 40:526-530.

10. Berg JS, Brunetti-Pierri N, Peters SU, Kang SH, Fong CT, Salamone J, Freedenberg D, Hannig VL, Prock LA, Miller DT, et al: Speech delay and autism spectrum behaviors are frequently associated with duplication of the 7q11.23 Williams-Beuren syndrome region. Genet Med 2007 9:427-441

11. Van der Aa N, Rooms L, Vandeweyer G, van den Ende J, Reyniers E, Fichera M, Romano C, Delle Chiaie B, Mortier G, Menten B, et al: Fourteen new cases contribute to the characterization of the $7 q 11.23$ microduplication syndrome. Eur J Med Genet 2009, 52:94-100.

12. Sbruzzi IC, Pereira AC, Vasconcelos B, Honjo RS, Krieger JE, Kim CA: Williams-Beuren syndrome: diagnosis by polymorphic markers. Genet Test Mol Biomarkers 2010, 14:209-214.
13. Dutra RL, Pieri PC, Teixeira AC, Honjo RS, Bertola DR, Kim CA: Detection of deletion at 7q11.23 in Williams-Beuren syndrome by polymorphic markers. Clinics (Sao Paulo) 2011, 66(6):959-964.

14. Schouten JP, McElgunn CJ, Waaijer R, Zwijnenburg D, Diepvens F, Pals G: Relative quantification of 40 nucleic acid sequences by multiplex ligation-dependent probe amplification. Nucleic Acids Res 2002, 30:e57.

15. van Hagen JM, Eussen HJ, van Schooten R, van Der Geest JN, Lagers-van Haselen GC, Wouters CH, De Zeeuw Cl, Gille JJ: Comparing two diagnostic laboratory tests for Williams syndrome: fluorescent in situ hybridization versus multiplex ligation-dependent probe amplification. Genet Test 2007, 11:321-327.

16. Karmiloff-Smith A, Grant J, Ewing S, Carette MJ, Metcalfe K, Donnai D, Read AP, Tassabehji M: Using case study comparisons to explore genotype-phenotype correlations in Williams-Beuren syndrome. J Med Genet 2003, 40:136-140

17. Pérez Jurado LA, Peoples R, Kaplan P, Hamel BC, Francke U: Molecular definition of the chromosome 7 deletion in Williams syndrome and parent-of-origin effects on growth. Am J Hum Genet 1996, 59:781-792.

18. Gilbert-Dussardier B, Bonneau D, Gigarel N, Le Merrer M, Bonnet D, Philip N, Serville F, Verloes A, Rossi A, Aymé S, et al: A novel microsatellite DNA marker at locus D7S1870 detects hemizygosity in 75\% of patients with Williams syndrome. Am J Hum Genet 1995, 56:542-544.

19. Brøndum-Nielsen K, Beck B, Gyftodimou J, Hørlyk H, Liljenberg U, Petersen MB, Pedersen W, Petersen MB, Sand A, Skovby F, et al: Investigation of deletions at 7q11.23 in 44 patients referred for WilliamsBeuren syndrome, using FISH and four DNA polymorphisms. Hum Genet 1997, 99:56-61.

20. Merla G, Brunetti-Pierri N, Micale L, Fusco C: Copy number variants at Williams-Beuren syndrome 7q11.23 region. Hum Genet 2010, 128(1):3-26.

21. Kozlowski P, Jasinska AJ, Kwiatkowski DJ: New applications and developments in the use of multiplex ligation-dependent probe amplification. Electrophoresis 2008, 29:4627-4636.

22. Bishop B: Applications of fluorescence in situ hybridization (FISH) in detecting genetic aberrations of medical significance. Biohorizons 2010, 3:85-95

23. Howald C, Merla G, Digilio MC, Amenta S, Lyle R, Deutsch S, Choudhury U, Bottani A, Antonarakis SE, Fryssira H, Dallapiccola B, Reymond A: Two high throughput technologies to detect segmental aneuploidies identify new Williams-Beuren syndrome patients with atypical deletions. J Med Genet 2006, 43:266-273.

24. Schubert C, Laccone F: Williams-Beuren syndrome: determination of deletion size using quantitative real-time PCR. Int J Mol Med 2006 18:799-806.

25. Snijders AM, Nowak N, Segraves R, Blackwood S, Brown N, Conroy J, Hamilton G, Hindle AK, Huey B, Kimura K, et al: Assembly of microarrays for genome-wide measurement of DNA copy number. Nat Genet 2011, 29:263-264.

26. Shaffer $L G$, Bejjani BA: A cytogeneticist's perspective on genomic microarrays. Hum Reprod Update 2004, 10:221-226.

27. Jehee FS, Takamori JT, Medeiros PF, Pordeus AC, Latini FR, Bertola DR, Kim CA, Passos-Bueno MR: Using a combination of MLPA kits to detect chromosomal imbalances in patients with multiple congenital anomalies and mental retardation is a valuable choice for developing countries. Eur J Med Genet 2011, 54(4):e425-e432.

28. Miller SA, Dykes DD, Polesky HF: A simple salting out procedure for extracting DNA from human nucleated cells. Nucleic Acids Res 1988 16:1215

doi:10.1186/1756-0500-5-13

Cite this article as: Dutra et al: Copy number variation in WilliamsBeuren syndrome: suitable diagnostic strategy for developing countries. BMC Research Notes 2012 5:13. 